\title{
Position Sensing Method for Linear Synchronous Motor Propelled High-Speed Train by Using Single Cross-Loop Inductive Cable and Three-Phase Receiving Antennas
}

\author{
Yan Sun, ${ }^{1}$ Chang-Young Lee, ${ }^{1,2 *}$ Young-Jae Han, ${ }^{2}$ \\ Jeong-Min Jo, ${ }^{2}$ Jin-Ho Lee, ${ }^{2}$ and Kwan-Sup Lee ${ }^{2}$ \\ ${ }^{1}$ University of Science and Technology (KRRI campus), \\ 176, Cheoldobangmulgwan-ro, Uiwang-si, Gyeonggi-do, 16105, Republic of Korea \\ ${ }^{2}$ Korea Railroad Research Institute, \\ 176, Cheoldobangmulgwan-ro, Uiwang-si, Gyeonggi-do, 16105, Republic of Korea
}

(Received February 6, 2017; accepted April 4, 2017)

Keywords: high-speed train, linear synchronous motor, position sensing, single cross-loop inductive cable

In this paper, we propose a position sensing method (PSM), in which a single cross-loop inductive cable and three-phase receiving antennas are used, for high-speed trains with the linear synchronous motor (LSM). The proposed PSM is considered to be capable of detecting the realtime relative position to control a train traveling at speeds of up to $1000 \mathrm{~km} / \mathrm{h}$. The cross-loop inductive cable and receiving antenna are modeled and simulated to determine the optimum antenna dimensions. The signal processing unit is also simulated to obtain position information from the induced voltage in the three receiving antennas. The PSM with the use of a single crossloop inductive cable and three-phase receiving antennas is tested by experiment, and it is found that the proposed PSM has great potential to detect the position of LSM-propelled $1000 \mathrm{~km} / \mathrm{h}$ highspeed trains.

\section{Introduction}

Because of its high power density and speed controllability, an air-core linear synchronous motor (LSM) with a superconducting electromagnet has been considered as a vehicle propulsion system from the high-speed maglev train to the Hyperloop, a currently conceptualized $1000 \mathrm{~km} / \mathrm{h}$ vacuum tube vehicle. ${ }^{(1,2)}$ To control the LSM, the precise relative position between the vehicle magnet and LSM armature coil must be known in real time. From the detected position, control of the speed and thrust of the LSM can be realized by adjusting the frequency and magnitude of current fed to the LSM armature coil. ${ }^{(3)}$

Several noncontact position-detecting technologies using optical, magnetic, and inductive methods are being used in industrial LSM applications. Among them, the inductive method is considered to be the most practical for high-speed trains with an air-core LSM. More details are introduced in Ref. 3. Several studies have been carried out on the inductive method using a crossloop inductive cable for the maglev system. ${ }^{(4-6)}$ An onboard excitation antenna and six-paired cross-loop inductive cables are used to detect the speed and position of a superconducting maglev

*Corresponding author: e-mail: cylee@krri.re.kr http://dx.doi.org/10.18494/SAM.2017.1579 
(SCmaglev) train, ${ }^{(7,8)}$ and the speed record of the SCmaglev train reached $603 \mathrm{~km} / \mathrm{h}$ on 21 April 2015. ${ }^{(9)}$ However, this method has constrains in high-speed application. When a vehicle travels at a speed of $1000 \mathrm{~km} / \mathrm{h}$, it might be difficult to detect the presence of an excitation antenna owing to the high speed. Even if an induced signal could be detected, the weak signal is sensitive to interference, making its detection a great challenge for the signal processing unit. Also, attenuation will occur when the induced signal is transmitted to the signal processing unit. Moreover, the six-paired cross-loop inductive cable is complicated and has a cross-talk interference problem. Therefore, it is necessary to propose a position sensing method (PSM) that is capable of detecting the position of a train moving at high speeds of up to $1000 \mathrm{~km} / \mathrm{h}$.

The rest of this paper is organized as follows. The concept design of the air-core LSM is briefly introduced and the importance of a position-sensing system for LSM control is explained. The feasibility of the proposed PSM for $1000 \mathrm{~km} / \mathrm{h}$ application is analyzed theoretically by comparison with the six-paired cross-loop inductive cable PSM. Optimum dimensions of the receiving antenna are designed by modeling and simulating the inductive loop cable and receiving antenna. The signal processing unit is also simulated to obtain position information from the induced signal in the three receiving antennas. The proposed PSM with a single cross-loop inductive cable and three-phase receiving antenna is tested by experiment. Conclusions are given in the final section.

\section{Concept Design of Air-Core LSM and Importance of Position Sensing System for LSM Control}

The LSM is a linear motor in which the mechanical motion is in synchrony with the magnetic field, i.e., the mechanical speed is the same as the speed of the travelling magnetic field. The propulsion force can be generated as an action of the travelling magnetic field produced by threephase winding and an array of magnetic poles $\mathrm{N}, \mathrm{S}, \ldots \mathrm{N}, \mathrm{S}$. The part producing the travelling magnetic field is called the armature. The part that provides the DC magnetic flux is called the field excitation system. ${ }^{(3,10)}$

Figure 1 shows the concept design of the air-core LSM for a $1000 \mathrm{~km} / \mathrm{h}$ high-speed train. As in Fig. 1(a), the air-core LSM consists of a three-phase armature coil laid on the ground to generate the travelling magnetic field and a superconducting electromagnet installed on the vehicle to produce DC magnetic flux. Figure 1(b) shows the cross-sectional view of the air-core LSM. The travelling magnetic field and linear motion of the magnetic field from the onboard superconducting electromagnet are synchronized, where the phase difference $\varphi$ is adjusted to ensure system efficiency, and $\tau$ is the pole pitch of the superconducting electromagnet. The motion of the superconducting electromagnet is controlled by current fed to the three-phase armature coil on the ground.

A speed and position sensing system is indispensable for traffic control, safety control, and drive control of a high-speed train. Real-time LSM control is needed to ensure that trains travel under the speed limits and in case of emergency. Drive control must switch the section switchgears on and off in accordance with the position of the traveling train, since the section power supply system for the LSM-propelled train is designed to reduce the energy consumption. ${ }^{(3)}$

\section{Theoretical Analysis of Proposed PSM for $1000 \mathrm{~km} / \mathrm{h}$ Application}

The air-core LSM is considered as a propulsion system for $1000 \mathrm{~km} / \mathrm{h}$ high-speed trains because of its high power density and speed controllability. The pole pitch of the LSM is important 


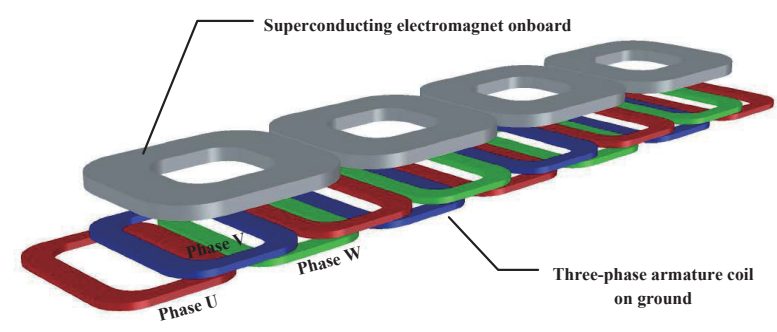

(a)

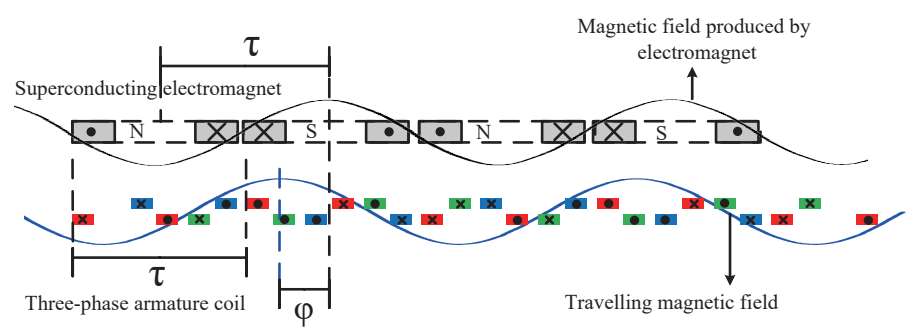

(b)

Fig. 1. Conceptual design of air-core LSM for $1000 \mathrm{~km} / \mathrm{h}$ high-speed train. superconducting electromagnet. (b) Cross-sectional view of air-core LSM.

(a) Air-core LSM with

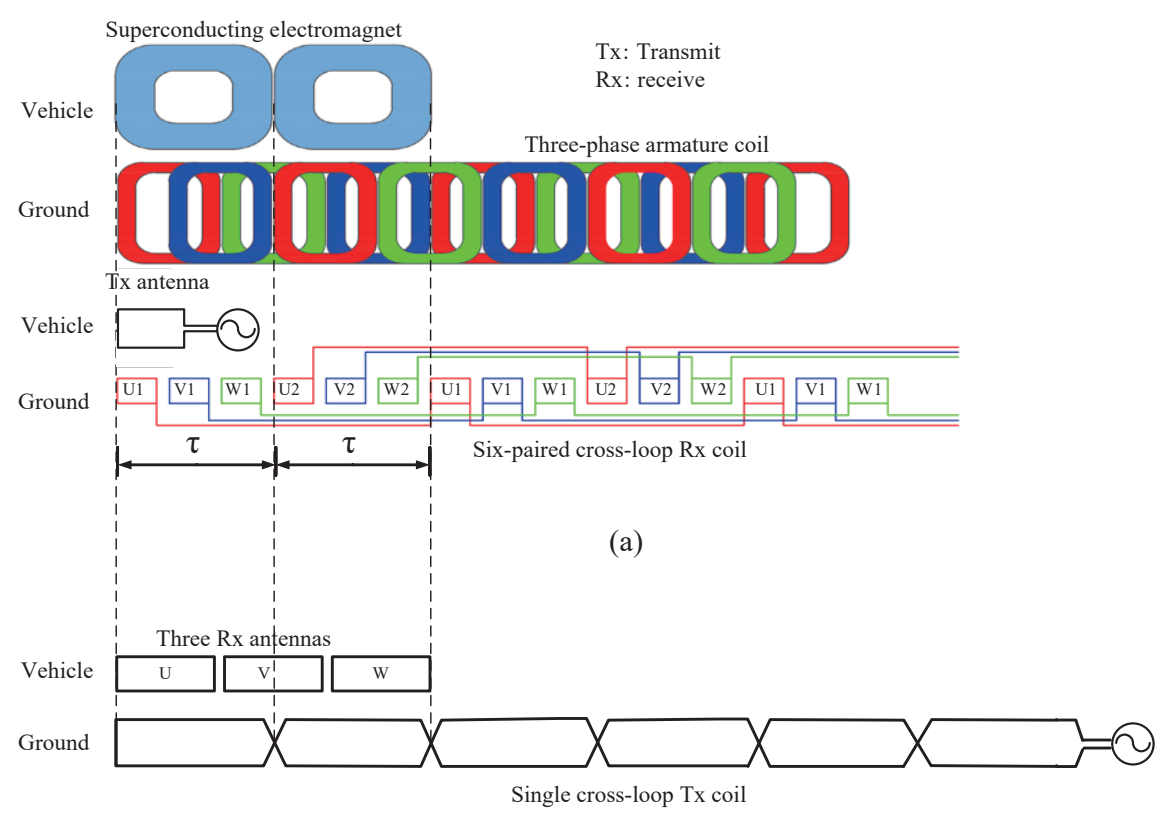

(b)

Fig. 2. Design of PSM for high-speed train in accordance with LSM configuration. (a) Six-paired cross-loop PSM. (b) Single cross-loop PSM.

in the PSM design since, with a fixed LSM configuration, the length of the periodically repeated cross-loop must be accordingly designed. The $1000 \mathrm{~km} / \mathrm{h}$ high-speed train has an air-core LSM design with a pole pitch of $600 \mathrm{~mm}$ and the SCmaglev LSM has a pole pitch of $1350 \mathrm{~mm} .{ }^{(3)}$ If the method with an onboard excitation antenna and six-paired cross-loop inductive cable used for the SCmaglev train is applied to detect the position of a $1000 \mathrm{~km} / \mathrm{h}$ high-speed train, it would be more difficult to detect the vehicle position because a smaller inductive loop is required to detect the position of a vehicle traveling at a much higher speed. Therefore, the PSM with a single cross-loop inductive cable and three-phase receiving antenna is proposed.

According to Faraday's law of electromagnetic induction, when high-frequency excitation is fed to a transmission coil, voltage can be induced at a receiving coil. For the six-paired cross-loop PSM in Fig. 2, the amplitude of the induced voltage at the six-paired cross-loop varies as the Tx 
antenna moves along the track. Moreover, for the single cross-loop PSM in Fig. 2, the amplitude of the induced voltage at three Rx antennas varies as they move along the Tx coil. At the signal processing unit, the envelope of variations must be demodulated. During this procedure, the number of excitation pulses is important because it affects the position sensing accuracy. The following equations describe the excitation pulse number for designs (a) and (b) in Fig. 2.

$$
\begin{aligned}
& \operatorname{Num}_{a}=\frac{2 \tau}{6 v_{a}} f_{a}, \\
& N_{u m}=\frac{2 \tau}{2 v_{b}} f_{b},
\end{aligned}
$$

where $\tau$ is the pole pitch, $v$ is the travelling speed, and $f$ is the excitation frequency.

Figure 2(a) shows the concept design of the six-paired cross-loop PSM. Equation (1) defines the number of excitation pulses of the six-paired cross-loop PSM. From Eq. (1), it can be seen that there are two methods of solving the problem due to high speed: one is to increase the excitation frequency $f_{a}$ and the other is to increase the pole pitch. However, as the pole pitch of the LSM is fixed, the only alternative is to increase the excitation frequency $f_{a}$ of the Tx antenna. Increased excitation frequency will result in a shorter section length of the Rx cable. Impedance matching and signal processing on the ground will also become complicated.

Figure 2(b) shows the concept design of the single cross-loop PSM. The single cross-loop cable is laid on the ground as the transmitter, and three identical antennas are loaded onboard as receivers. One loop of the ground cable covers one pole pitch. The three antennas are arranged to equally cover two pole pitches. When the excitation with the frequency $f$ is fed to the Tx coil, the induced voltages of three Rx antennas can be expressed as in Eqs. (3)-(5), ${ }^{(11)}$ because a timechanging magnetic field is generated in accordance with the Tx cross-loop coil as the vehicle travels along the track. After signal processing, the three-phase signal with $120^{\circ}$ phase differences can be obtained, as described by Eqs. (6)-(8). From the three-phase signal, phase information $\theta$ can be derived, as in Eq. (9). Since $\theta$ increases linearly with $2 \pi$ when the travelling distance is increased by $2 \tau, \theta$ can be used to measure the vehicle position continuously, as shown in Fig. 3. Therefore, the precise vehicle speed and position can be continuously monitored. ${ }^{(11,12)}$

$$
\begin{gathered}
E_{\text {ind_u }}=\cos \left(2 \pi \frac{x}{2 \tau}\right) \times \cos (2 \pi f t) \\
E_{\text {ind_v }}=\cos \left(2 \pi \frac{x}{2 \tau}-\frac{2 \pi}{3}\right) \times \cos (2 \pi f t) \\
E_{\text {ind_w }}=\cos \left(2 \pi \frac{x}{2 \tau}+\frac{2 \pi}{3}\right) \times \cos (2 \pi f t) \\
E_{u}=\cos \left(2 \pi \frac{x}{2 \tau}\right) \\
E_{v}=\cos \left(2 \pi \frac{x}{2 \tau}-\frac{2 \pi}{3}\right)
\end{gathered}
$$




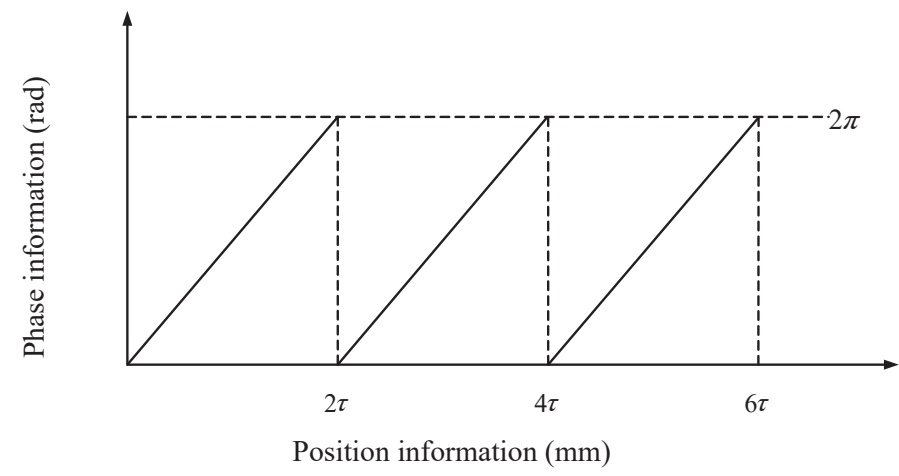

Fig. 3. Relationship between position information and phase information.

$$
\begin{gathered}
E_{w}=\cos \left(2 \pi \frac{x}{2 \tau}+\frac{2 \pi}{3}\right) \\
\theta=2 \pi \frac{x}{2 \tau}=\tan ^{-1} \frac{\sqrt{3}\left(E_{v}-E_{w}\right)}{2 E_{u}-\left(E_{v}+E_{w}\right)} \\
\frac{\theta}{2 \pi}=\frac{x}{2 \tau}
\end{gathered}
$$

There are advantages to the proposed PSM. On the transmission side, only a single cross-loop cable, which is simple and cross-talk-free compared with the six-paired cross-loop, is needed. The signal process unit simultaneously deals with the received signal from three Rx antennas without the need to consider attenuation, while attenuation must be considered for the six-paired cross-loop PSM. Although there would be a delay problem to the transfer of processed position information to the control center, it can be solved at the wireless communication unit. Equation (2) defines the number of excitation pulses of the single cross-loop PSM. Under the same condition, $f_{a}=f_{b}$ and $v_{a}=v_{b}$, the number of excitation pulses of the single cross-loop PSM is three times that of the sixpaired cross-loop PSM by comparing Eqs. (1) and (2). However, in design (b) of Fig. 2, even the train speed is increased by three times, and the same number of excitation pulses can be calculated without increasing excitation frequency.

\section{Modeling, Simulation, and Experiment}

\subsection{Modeling and simulation of single cross-loop cable and receiving antenna}

In accordance with the design in Fig. 2(b), a simplified model of the cross-loop cable and $\mathrm{Rx}$ antenna is made, as shown in Fig. 4. Tx represents one loop of the cross-loop cable and Rx represents one of the three receiving antennas.

Excitation is fed to the Tx loop and induced voltage can be detected at the Rx antenna as it moves. Because of design limitations, the length of the Tx loop and the pole pitch are both 600 


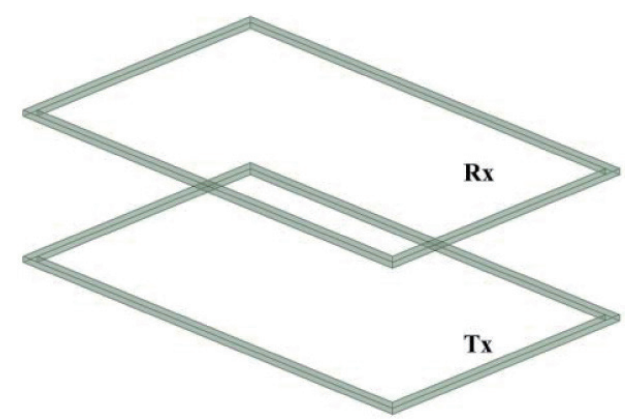

Fig. 4. Simplified model of single cross-loop PSM.
Table 1

Design variables for simulation.

\begin{tabular}{lrrrr}
\hline Variable & \multicolumn{4}{c}{ Value } \\
\hline Length (mm) & 250 & 300 & 350 & 400 \\
Width (mm) & 60 & 80 & 100 & 120 \\
Gap (mm) & 50 & 100 & 150 & 200 \\
Amplitude (V) & 5 & 10 & - & - \\
\hline
\end{tabular}

$\mathrm{mm}$, and the width of the Tx loop is set to $120 \mathrm{~mm}$. In order to avoid interference, the maximum length of the Rx antenna is $400 \mathrm{~mm}$, which is one-third of twice the pole pitch. In order to elucidate the optimum design of the Rx antenna, various values of the design variables of the length and width of the Rx antenna, as listed in Table 1, and other variables such as excitation amplitude and gap distance, were used in the simulation.

The induced voltage for different design variables is calculated according to the principle of Faraday's law. The calculation is carried out by Maxwell, a commercial finite element method (FEM) tool. Figure 5 shows the simulation results using the design variables. From Figs. 5(a) and 5(b), we can see that, within the design limitation, the optimum size of the Rx antenna is $400 \mathrm{~mm}$ in length and $120 \mathrm{~mm}$ in width. Figure 5(c) indicates that induced voltage decreased rapidly as gap distance increased. Figure 5(d) is the simulation result of the induced voltage of the Rx antenna at different positions along the track direction. The result is the same as the expectation: the largest value is induced at the center position and values are smaller as the Rx antenna moves away from the center. Also, a large induced voltage can be detected if strong excitation is fed to the Tx loop.

\subsection{Simulation of signal processing unit}

Simulation is carried out to obtain phase information using the algorithm described in Sect. 3. Figure 6 shows a block diagram of the signal processing unit. An ideal three-phase induced voltage signal is generated, and after demodulation and filtering, an envelope of induced voltage can be obtained. These three-phase signals are processed by the algorithm unit, and then phase information can be calculated.

Figure 7 shows the simulation result of the signal processing unit. Figure 7(a) shows the simulation result of the ideal induced voltage at three Rx antennas, and Fig. 7(b) is the envelope of induced voltage after demodulation and filtering. Figure 7(c) shows the relationship of the traveling time and phase information. In Eq. (10), the position information $x$ can be expressed as $x$ $=v t$, where $v$ is the vehicle speed. Thus, the relationship between the traveling time and the phase information can be derived as in Eq. (11).

$$
\theta=\frac{v \pi}{\tau} t
$$




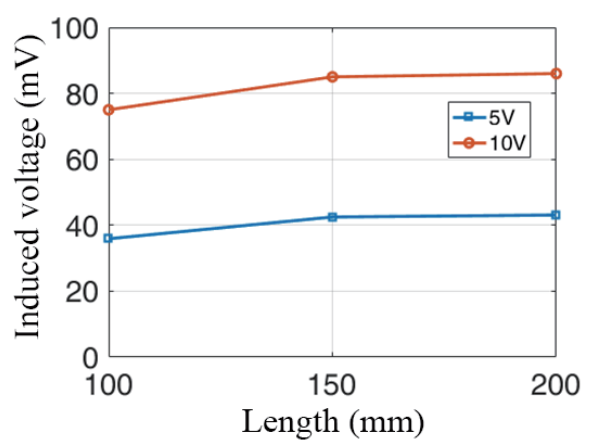

(a)

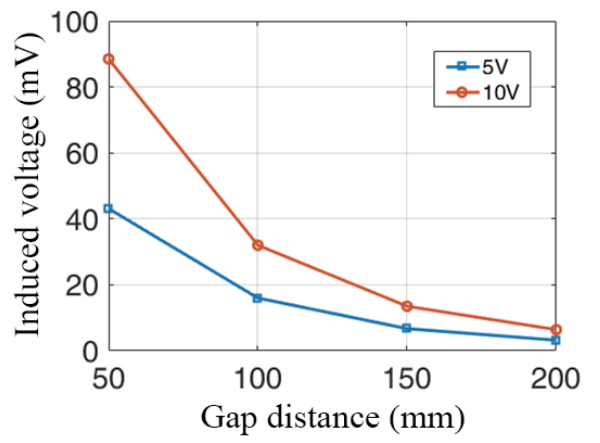

(c)

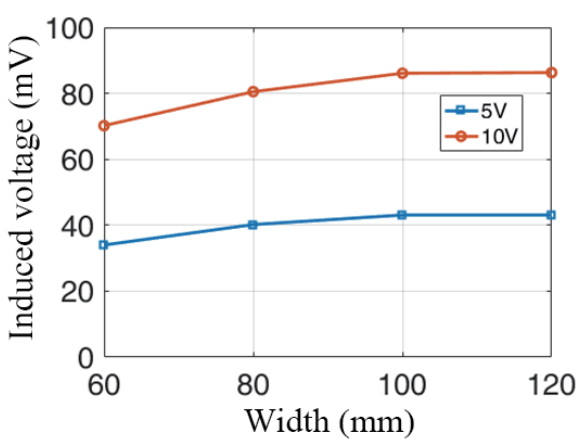

(b)

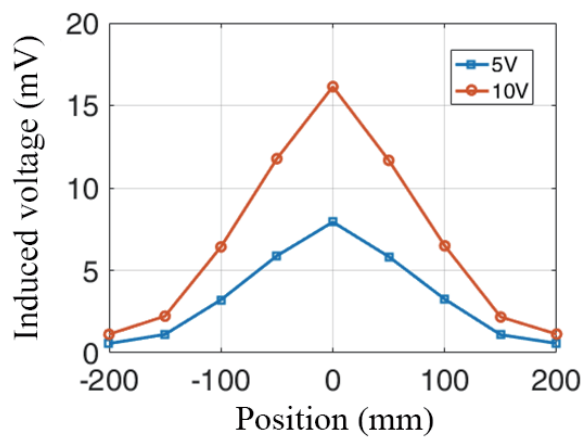

(d)

Fig. 5. (Color online) Simulation results for various variables. (a) Rx antenna length, (b) Rx antenna width, (c) Gap distance between Tx and Rx, and (d) Rx loop position.

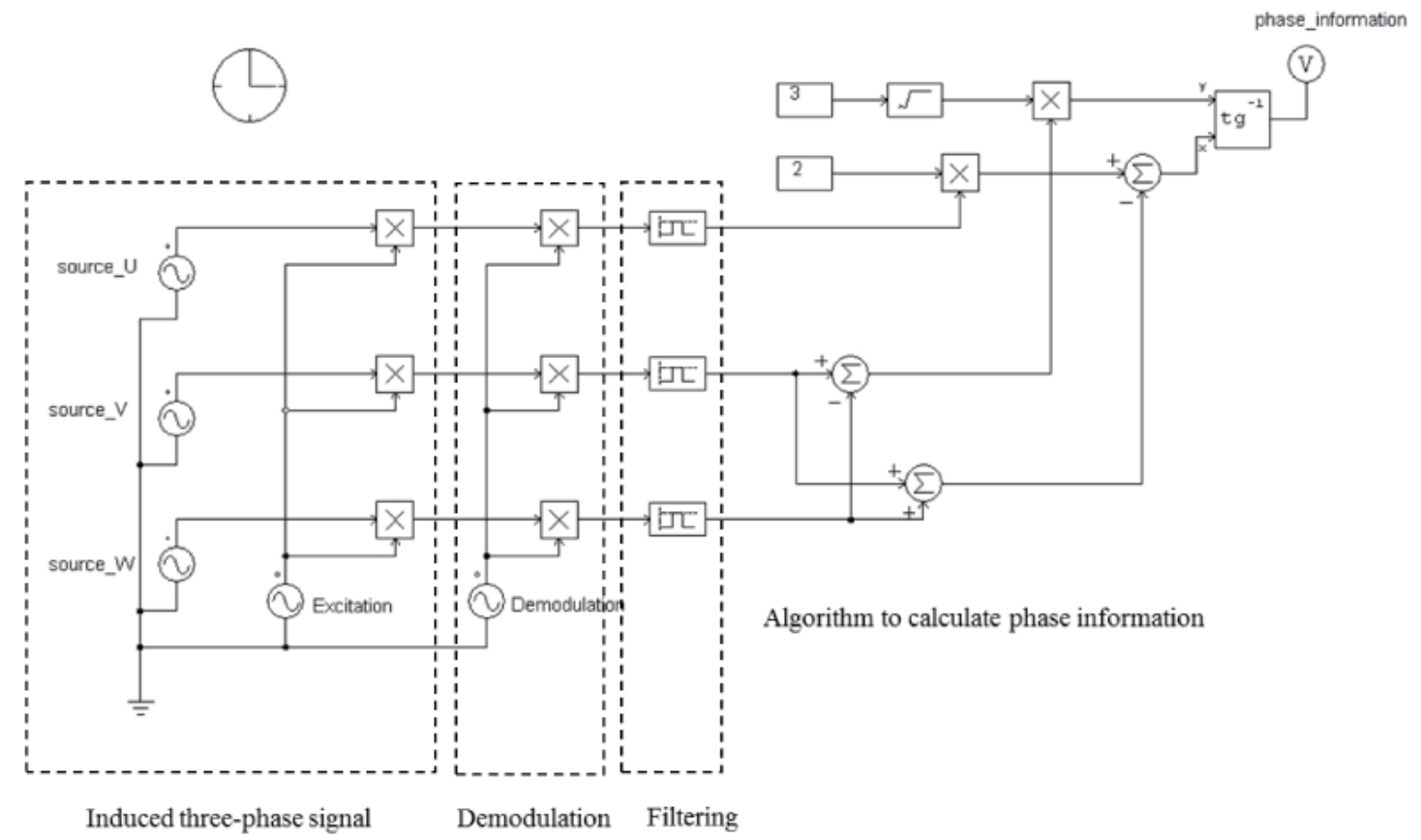

Fig. 6. Block diagram of signal processing unit. 


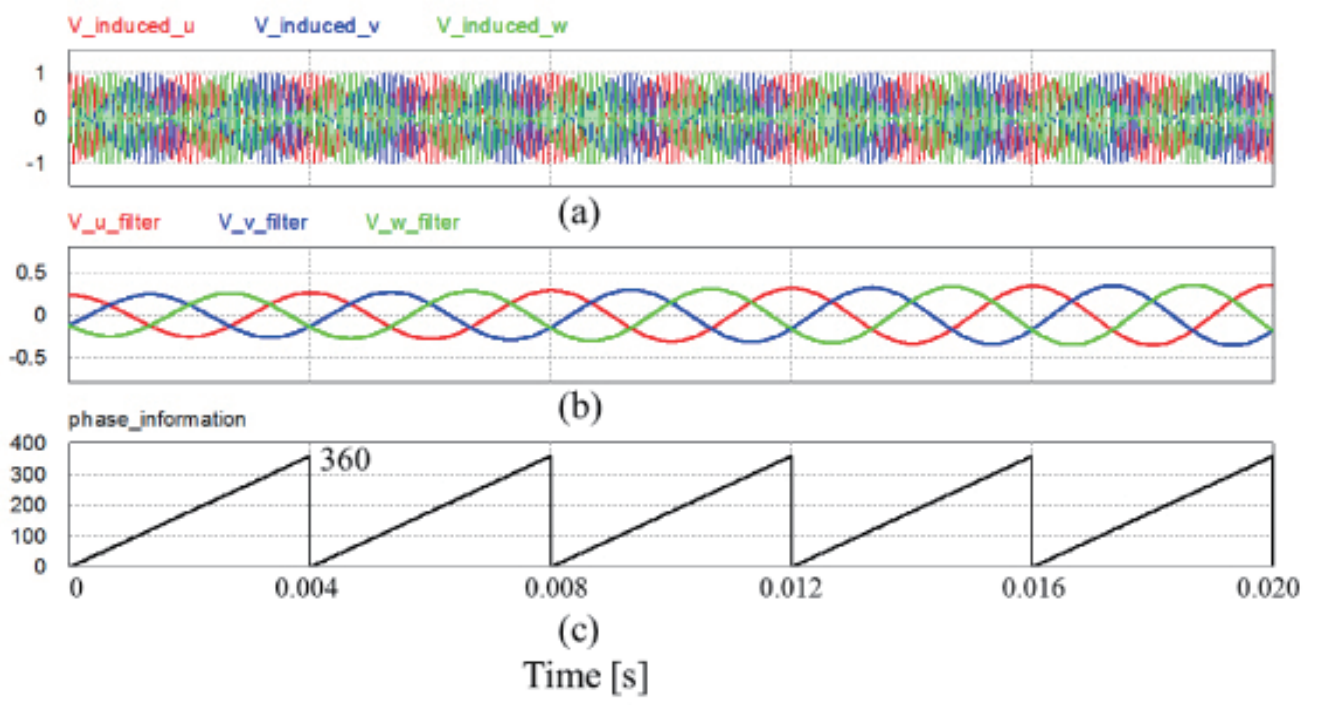

Fig. 7. (Color online) Simulation result of signal processing unit.

In this simulation, the vehicle speed $v$ is set to $1080 \mathrm{~km} / \mathrm{h}$, so the envelop frequency of the induced voltage is $250 \mathrm{~Hz}$. In the time of $0.004 \mathrm{~s}$, the vehicle moves a distance of $2 \tau$. Since the travelling distance and phase information are linearly related in the time interval of $0.004 \mathrm{~s}$, realtime position information can be obtained from phase information. From Fig. 7(c), real-time train speed can be derived as well, because the slope of the line and the train speed are related as shown in Eq. (12).

$$
\text { Line slope }=\frac{v \pi}{\tau}
$$

\subsection{Experiment of proposed PSM}

Figure 8 shows the experimental setup of the single cross-loop PSM. Design variables are the same as those in the simulation, as listed in Table 1.

The experiment result of induced voltage in the three Rx antennas is shown in Fig. 9. The value of induced voltage changes with different design variables. The speed in the experiment is much lower than $1000 \mathrm{~km} / \mathrm{h}$, and the received voltage is well detected. Further research is needed for high-speed experiments.

The experimental results of induced voltage using different variables are shown in Fig. 10. From the experimental results in Figs. 10(a)-10(c), the same conclusion can be reached as from the simulation result. Figure 10(d) shows the experimental result of induced voltage with excitation frequency as the design variable. We can see that an optimum excitation frequency exists and maximum voltage is induced at a frequency of around $1.2 \mathrm{MHz}$. The existence of an optimum frequency proved the importance of impedance matching. The simulation and experimental results have different induced voltages because the experiment setup cannot be exactly the same as the simulation. 


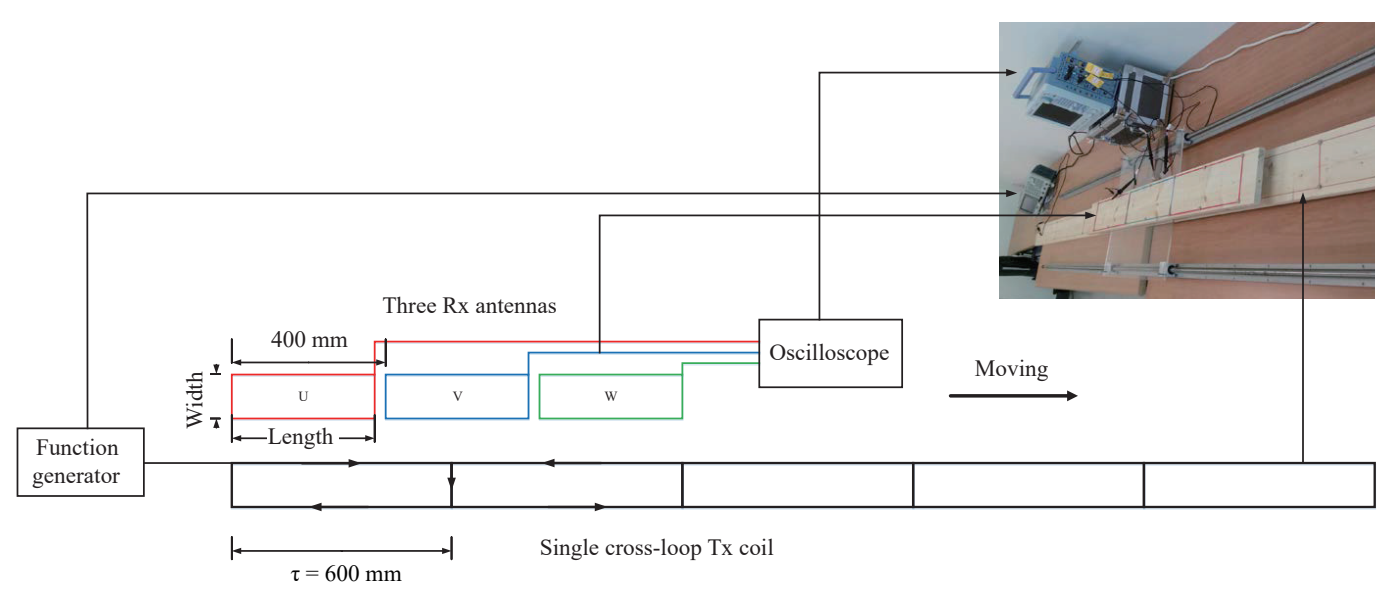

Fig. 8. (Color online) Experiment setup of proposed single cross-loop PSM.

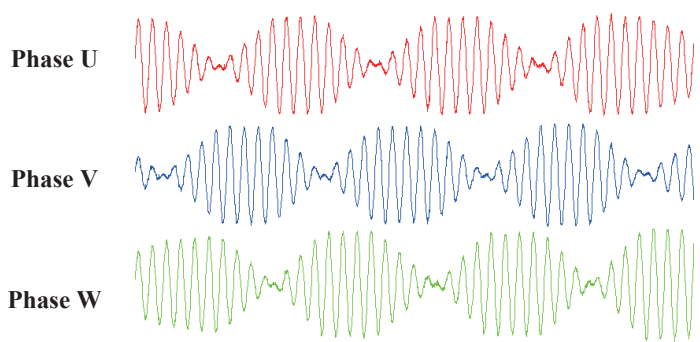

Fig. 9. (Color online) Induced voltage of three Rx antennas from experiments.

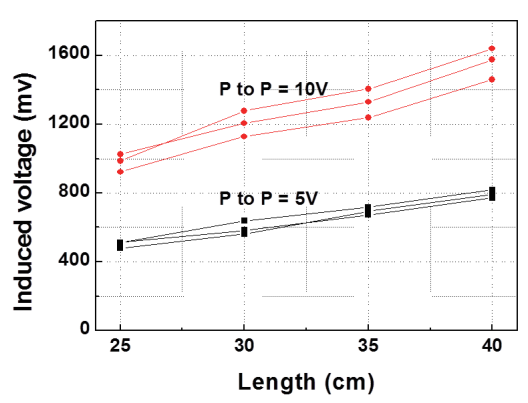

(a)

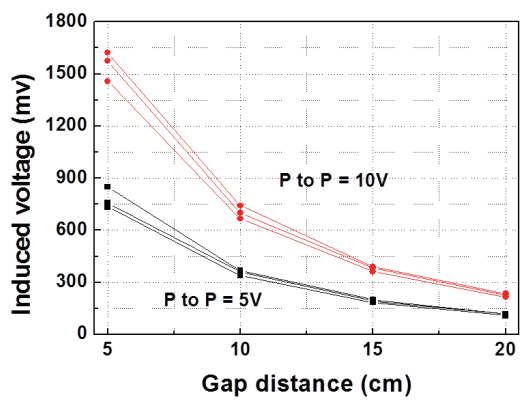

(c)

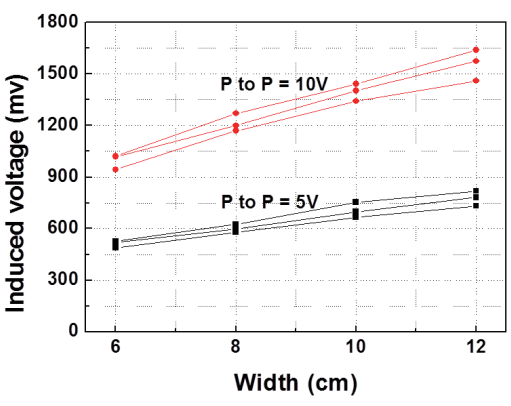

(b)

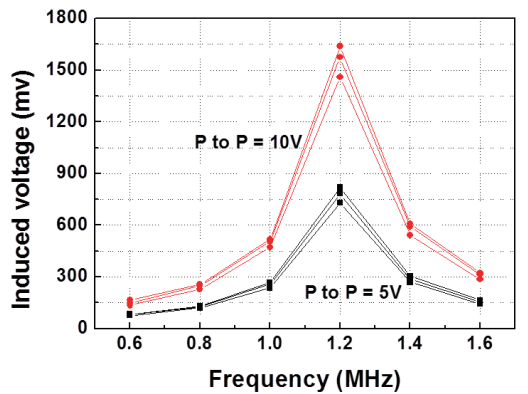

(d)

Fig. 10. (Color online) Experimental results for various design variables. (a) Rx antenna length, (b) Rx antenna width, (c) gap distance between Tx and Rx, and (d) excitation frequency. 


\section{Conclusions}

The position-detecting system is indispensable for realizing precise LSM control and ensuring safe operation for high-speed trains. A single cross-loop inductive cable PSM is proposed for $1000 \mathrm{~km} / \mathrm{h}$ high-speed trains with an air-core LSM propulsion system. When a vehicle travels at high speed, the six-paired cross-loop PSM is restricted by loop size, and other problems will arise if excitation frequency increases. However, the proposed single cross-loop PSM is capable of realizing high-speed detection with a simpler design and there is no need to consider crosstalk interference and attenuation. Modeling and simulation are carried out to design the Rx antenna, and experiment results are consistent with simulation results. Phase information can be calculated using a specific algorithm, and position information can be obtained from phase information. There are some other considerations when the proposed single cross-loop PSM is applied to a fullscale high-speed train system. Further research will focus on impedance matching, high-speed experiments, and wireless data transmission. Interference from the magnetic field generated by the LSM propulsion coil and superconducting electromagnet will be studied as well.

\section{Acknowledgements}

This research was supported by "Core technology development of subsonic capsule train" of the Korea Railroad Research Institute, Republic of Korea.

\section{References}

1 Wikipedia: Hyperloop, https://en.wikipedia.org/wiki/Hyperloop.

2 Space X: Hyperloop Alpha (2013).

3 J. Gieras, Z. Piech, and B. Tomczuk: Linear Synchronous Motors-Transportation and Automation Systems (CRC Press, 2000) 2nd ed. Chaps. 1, 5, and 6.

4 C. H. Dai, F. S. Dou, X. L. Song, and Z. Q. Long: Sensors 12 (2012) 8526.

5 F. Dou, X. Song, and C. Dai: Przeglad Elektrotechniczny R. 89 (2013) 140.

6 Y. Wang, J. S. Lian, and K. L. Zhang: Electr. Drive Locomotive 2 (2002) 33.

7 T. Sasaki: Electr. Eng. Jpn. 100 (1980) 219.

8 M. Ono, M. Ohta, K. Tazumi, and K. Mita: Int. Wire \& Cable Symp. Proc. (1997) p. 720.

9 J. McCurry: The Guardian, https://www.theguardian.com/world/2015/apr/21/japans-maglev-train-notches-upnew-world-speed-record-in-test-run (April 2016).

10 H. W. Lee, K. C. Kim, and J. Lee: IEEE Trans. Magn. 42 (2006) 1917.

11 E. Itakura and T. Sasaki: Electr. Eng. Jpn. 95 (1975) 115.

12 T. Hatta, T. Kusakabe, and T. Asai: Electr. Eng. Jpn. 106 (1986) 125. 